\title{
Mirogabalin treatment of postoperative neuropathic pain after thoracic surgery: study protocol for a multicenter, randomized, open-label, parallel-group, interventional trial
}

\author{
Ryoichiro Doi ${ }^{1 \#}$, Takuro Miyazaki ${ }^{1 \#}$, Tomoshi Tsuchiya ${ }^{1}$, Keitaro Matsumoto ${ }^{1}$, Koichi Tomoshige ${ }^{1}$, \\ Ryusuke Machino ${ }^{1}$, Satoshi Mizoguchi ${ }^{1}$, Takamune Matsumoto ${ }^{1}$, Keita Yamaguchi ${ }^{2}$, Hiroshi Takatsuna ${ }^{3}$, \\ Kazuhito Shiosakai ${ }^{4}$, Takeshi Nagayasu ${ }^{1}$ \\ ${ }^{1}$ Department of Surgical Oncology, Nagasaki University Graduate School of Biomedical Sciences, Nagasaki, Japan; ${ }^{2}$ Oncology Medical Science \\ Department, Daiichi Sankyo Co., Ltd., Tokyo, Japan; ${ }^{3}$ Primary Medical Science Department, Daiichi Sankyo Co., Ltd., Tokyo, Japan; ${ }^{4}$ Data \\ Intelligence Department, Daiichi Sankyo Co., Ltd., Tokyo, Japan \\ \#These authors contributed equally to this work. \\ Correspondence to: Takeshi Nagayasu, MD, PhD. Department of Surgical Oncology, Nagasaki University Graduate School of Biomedical Sciences, \\ 1-7-1 Sakamoto, Nagasaki 852-8501, Japan. Email: nagayasu@nagasaki-u.ac.jp.
}

Background: Intercostal nerve damage due to thoracotomy or thoracoscopic manipulation is a major contributor to chronic postsurgical pain after pulmonary resection. Chronic postsurgical pain may last for months or years and can negatively impair physical functioning and daily activities. Global consensus on severe postoperative pain management is lacking, and chronic pain incidence after thoracic surgery remains high. Many patients report neuropathic pain, which can be difficult to treat with currently available therapies. The efficacy and safety of mirogabalin have been demonstrated for other types of neuropathic pain; thus, this study was planned to investigate the efficacy and safety of mirogabalin to treat neuropathic pain after thoracic surgery.

Methods: In this multicenter, randomized, open-label, parallel-group, interventional study, patients who are diagnosed with neuropathic pain following removal of a chest drain after lung resection will receive conventional therapy (non-steroidal anti-inflammatory drugs and/or acetaminophen) with or without the addition of a clinical dose of mirogabalin for 8 weeks. For patient stratification, a visual analog scale pain intensity score at baseline of $<60 v s . \geq 60 \mathrm{~mm}$ will be used. Treatment efficacy and safety with and without the addition of mirogabalin will be assessed using a questionnaire evaluating postoperative changes in pain severity and activity. The primary study endpoint is the change in pain intensity from baseline to Week 8 , measured by the visual analog scale. Additionally, the presence of chronic pain at 12 weeks after enrollment in each treatment group will be recorded.

Discussion: This protocol has been reviewed and approved by the Clinical Research Review Board of Nagasaki University. Study data will be published in the Japan Registry of Clinical Trials database and peerreviewed journals. Mirogabalin is already approved for the treatment of other types of neuropathic pain. It is anticipated that this study will provide data to elucidate the impact of mirogabalin treatment, in combination with conventional therapy, to benefit patients with neuropathic pain following thoracic surgery.

Trial Registration: Japan Registry of Clinical Trials Identifier: jRCTs071200053.

Keywords: Mirogabalin; postoperative neuropathic pain; thoracic surgery; postthoracotomy pain syndrome

Submitted Apr 28, 2021. Accepted for publication Aug 19, 2021.

doi: $10.21037 /$ jtd-21-741

View this article at: https://dx.doi.org/10.21037/jtd-21-741 


\section{Introduction}

One of the most common reasons for visiting a hospital in the US is chronic pain (1-3). It is estimated that about $22.5 \%$ of cases involve pain that arises after surgery (4). Chronic postsurgical pain (CPSP) is defined as pain that continues for $\geq 3$ months following the surgical procedure $(5,6)$. In a meta-analysis, the incidence of chronic pain at 3 and 6 months after thoracic surgery was found to be $57 \%$ and $47 \%$, respectively (7). For some patients, CPSP may last many months or even years (8). Even mild pain, if it is persistent and chronic in nature, can impair physical function and reduce physical and social activities (9). Neuropathic pain is defined as pain resulting directly from a lesion or disease affecting the somatosensory system either at the peripheral or central level (10). Roughly a third of patients who develop CPSP after thoracic surgery present a neuropathic pain component, which has been associated with more marked physical dysfunction, as well as worse quality of life, than CPSP without the neuropathic component (11).

Intercostal nerve damage due to thoracotomy or thoracoscopic manipulation is a major contributor to CPSP after pulmonary resection (12), with variations in incidence depending on the types of surgical procedures and manipulations performed $(13,14)$. In a retrospective analysis of thoracic surgery in Japan, $10 / 14$ patients (71.4\%) who converted from minimally invasive videoassisted thoracoscopic surgery to invasive thoracotomy reported postoperative neuropathic pain. Of these, three patients developed intractable symptoms (15). In a second retrospective analysis of 200 Italian patients who underwent lung resection via minithoracotomy or videoassisted thoracoscopic surgery, the overall incidence of CPSP was $35 \%$. Additionally, pain with a neuropathic component occurred at an incidence of $16 \%$ in women and $6 \%$ in men (16). A numeric rating scale ranging from $0-10$ can be used in clinical settings to assess the severity of chronic pain related to thoracic surgery (17). Studies using this reliable and validated measure suggest that pain control during the acute postoperative phase and for up to 3 months following surgery is critical for reducing CPSP (17). However, despite the high rate of chronic pain development after thoracic surgery $(11,18)$, optimal pain treatments during the first 3 postoperative months have not been established.

Current treatments for pain control after thoracic surgery include local anesthetics (epidural anesthesia, paravertebral body block, intercostal nerve block), which are commonly administered in the perioperative period (19). Oral anti-inflammatory analgesics [e.g., nonsteroidal anti-inflammatory drugs (NSAIDs) and/or acetaminophen] can also be administered postoperatively and have been shown to be beneficial in postoperative surgical patients without contraindications (20). For severe postoperative pain, treatments vary by region, and consensus on a global management protocol is lacking. In Japan, antiepileptic drugs or oral agents to treat neuropathic pain may be prescribed (21); elsewhere, narcotic agents or multidisciplinary management techniques are recommended (22). Recommended firstline drugs for the treatment of neuropathic pain include tricyclic antidepressants, serotonin-noradrenaline reuptake inhibitors (e.g., duloxetine), and gabapentinoids (e.g., gabapentin and pregabalin) $(23,24)$. However, clinical outcomes have varied; pregabalin has shown some effectiveness in reducing pain after thoracic surgery $(25,26)$ but lacked efficacy during the critical early postoperative stage (27). Overall, despite treatment, it is clear that chronic pain incidence after thoracic surgery remains high.

Mirogabalin besilate (hereinafter referred to as mirogabalin) is approved in Japan as a treatment for peripheral neuropathic pain $(28,29)$, and provides analgesic effects via binding to the $\alpha_{2} \delta$ subunit of voltage-gated calcium channels (30). The efficacy and safety of mirogabalin have been demonstrated for other types of neuropathic pain, such as diabetic peripheral neuropathic pain (31-33) and postherpetic neuralgia $(34,35)$. Mirogabalin has not yet been assessed in patients with thoracic postsurgical pain, in whom the incidence of neuropathic pain is also high. However, it is anticipated that mirogabalin treatment will benefit patients with neuropathic pain following thoracic surgery.

We present the following article in accordance with the SPIRIT reporting checklist (available at https://dx.doi. org/10.21037/jtd-21-741).

\section{Research purpose}

The study is being conducted in accordance with the Declaration of Helsinki (as revised in 2013). The study was approved by the Clinical Research Review Board of Nagasaki University (approval number CRB7180001), and informed consent will be obtained from all individual participants.

This study was planned to investigate the efficacy and 


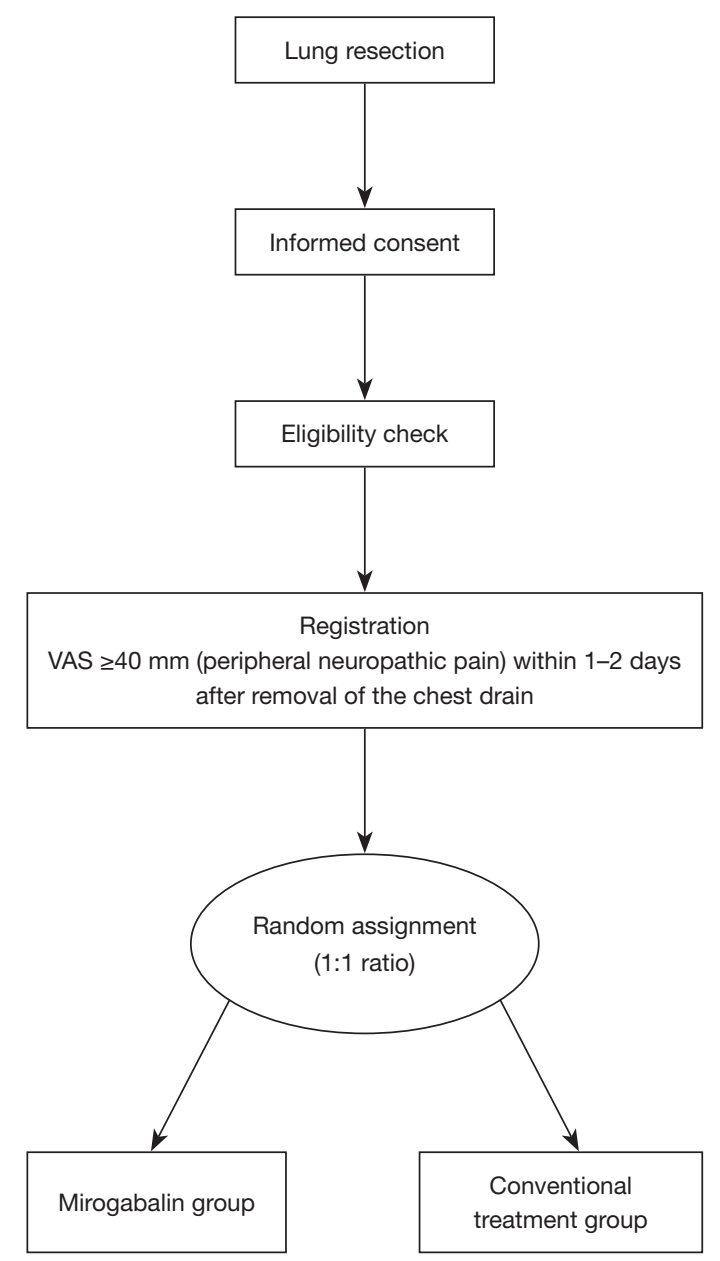

Figure 1 Study flow. Patients assigned to the conventional treatment group received conventional pain medications (nonsteroidal anti-inflammatory drugs and/or acetaminophen). Patients assigned to the mirogabalin group received conventional treatment plus mirogabalin. VAS, visual analog scale.

safety of mirogabalin, in combination with conventional therapy, in treating neuropathic pain after thoracic surgery. As a chest tube insertion has deleterious effects on intercostal nerve function (14), patients who undergo lung resection will receive a clinical dose of mirogabalin in addition to conventional therapy for 8 weeks if they are diagnosed with neuropathic pain after the removal of the chest drain. This will be applied regardless of the type of surgical resection. Treatment efficacy and safety with and without the addition of mirogabalin will be assessed based on the results of a questionnaire evaluating postoperative changes in pain severity and activity. In addition, the presence of chronic pain at 12 weeks after enrollment in each treatment group will be recorded.

The study is registered at the Japan Registry of Clinical Trials (jRCT) with the identifier jRCTs071200053.

\section{Study details}

\section{Study design and etbical considerations}

This is a multicenter, randomized, open-label, parallelgroup, interventional study. Participation of patients from at least 14 medical institutions is planned (Table S1), and a schematic of the study flow is provided in Figure 1. The enrollment period began in December 2020 and will remain open until December 2021; the observation period will run from December 2020 to March 2022.

The study is being conducted in accordance with the Declaration of Helsinki (revised October 2013) and the Clinical Research Act (promulgated April 14, 2017). In addition, all applicable local, national, and international legislation will be applied. The study protocol and associated documentation have been reviewed and approved by the Clinical Research Review Board of Nagasaki University (approval number CRB7180001), and permission to conduct the study was obtained from the administrators of each participating medical organization. The protocol is designated TLG-DS-19009 (version 3.0; dated March 25, 2021).

\section{Participant selection}

In principle, all patients undergoing lung resection at the participating medical institutions will be screened for study eligibility, with application of the detailed inclusion and exclusion criteria following the surgical procedure to identify patients with neuropathic postoperative pain suitable for study participation.

The inclusion criteria are as follows: patients undergoing lung resection (for any medical condition) who were aged $\geq 20$ years at the time of informed consent; study enrollment was possible within 1-2 days after removal of the chest drain at the time of pulmonary resection; a visual analog scale (VAS) score of $\geq 40 \mathrm{~mm}$ (range $0-100 \mathrm{~mm}$ where $0 \mathrm{~mm}$ is no pain and $100 \mathrm{~mm}$ is worst pain) for perioperative pain at rest at the time of enrollment; hypoesthesia under the innervation of the intercostal space at the wound site (to exclude postoperative pain mainly caused by nociceptive 
pain and to restrict eligibility to neuropathic pain) and no residual effect of epidural anesthesia at enrollment. All patients are required to provide written informed consent (documented by the study investigator) prior to study participation.

To ensure that patients with peripheral neuropathy after thoracic surgery are correctly and consistently diagnosed, neuropathic pain will be evaluated using a neuropathic pain diagnostic algorithm for subjective symptoms that includes a questionnaire and a pin-prick sensation test as an objective assessment of symptoms based on the grading system developed by the International Association for the Study of Pain Special Interest Group on Neuropathic Pain (36). The test for loss of pin-prick sensation will be performed at registration. In brief, patients will be placed supine with their eyes closed and normal sensation outside the site of surgery will be tested using a toothpick. It will be confirmed that there are no effects due to anesthesia (if anesthesia is determined to affect the results, the test will be repeated at a later time point). Next, hypoesthesia at the surgical wound site will be determined; the presence of hypoesthesia is taken to indicate neuropathy.

The exclusion criteria include total pleuropulmonary resection or pleurectomy; history of previous thoracotomy or thoracoscopic surgery resulting in neuropathy which continued until the time of the current surgery (and which would confound the identification of neuropathy resulting from the most recent surgery); serious liver dysfunction at enrollment; creatinine clearance (Cockcroft-Gault equation) $<30 \mathrm{~mL} / \mathrm{min}$ in the 3 months prior to enrollment; use of medications for neuropathic pain between 1 month before surgery and the time of enrollment; receipt of neoadjuvant chemotherapy within 2 months before surgery (to exclude chemotherapy-related neuropathic pain); hypersensitivity to any study treatment; pregnancy or lactation; presence of severe pain outside the perioperative wound area complicating the assessment of efficacy in this study; and any patient deemed inappropriate for participation in the study by the investigator or who might be endangered by study participation. If any patients were to require adjuvant chemotherapy with cisplatin, such patients would be discontinued from the study.

The concomitant use of several therapies will be prohibited during the study period. These therapies include pregabalin and gabapentin, duloxetine, tramadol, platinum chemotherapy agents, probenecid and cimetidine, and lorazepam. Postoperative nerve block, surgical procedures, or any other intervention (e.g., electrical stimulation, radiation therapy) that may affect the evaluation of treatment effectiveness will also be prohibited.

\section{Randomization and interventions}

Eligible patients will be randomly assigned to one of two treatment groups by the registration system, using a permuted block method (ratio 1:1). The stratification factors used in this study will be a VAS score of $<60 \mathrm{vs}$. $\geq 60 \mathrm{~mm}$ at baseline and study site.

In the conventional treatment group, NSAIDs and/ or acetaminophen will be prescribed per usual practice and in accordance with the package insert (including ondemand use) and insurance coverage. Patients are required to maintain a stable treatment regimen during the study. If the given medication does not adequately control pain, the investigator will consider prescribing medications that are not listed among the prohibited concomitant medications. For patients in the conventional treatment group, the investigator will consider increasing the dose of concomitant drugs to treat pain if needed to achieve pain control. Nevertheless, patient pain control will prevail as per ethical standards. If patients require prohibited concomitant medications to manage their pain, they will be discontinued from the study.

In the mirogabalin treatment group, in addition to conventional treatment, patients will also receive mirogabalin for 8 weeks. The dosage will be adjusted according to creatinine clearance, in line with the package insert. For patients with creatinine clearance $\geq 60 \mathrm{~mL} / \mathrm{min}$, the mirogabalin dose administered will be $5 \mathrm{mg}$ twice daily (BID) during Week 1, $10 \mathrm{mg}$ BID during Week 2, and $15 \mathrm{mg}$ BID thereafter. Additional adjustments are allowed for tolerability, between $10-15 \mathrm{mg}$ BID. For patients with creatinine clearance $\geq 30$ and $<60 \mathrm{~mL} / \mathrm{min}$, the mirogabalin dose administered will be $2.5 \mathrm{mg}$ BID during Week $1,5 \mathrm{mg}$ BID during Week 2, and $7.5 \mathrm{mg}$ BID thereafter. Additional adjustments are allowed for tolerability, between $5-7.5 \mathrm{mg}$ BID. In cases where mirogabalin is discontinued, caution should be taken, and a gradual taper implemented. Patients who discontinue mirogabalin within 8 weeks from the start of treatment will be discontinued from the study.

\section{Measures and endpoints}

The study schedule is shown in Table 1. Data on baseline patient, surgical, and treatment characteristics will be collected. Additionally, the following items will be 
Table 1 Investigation, observation, examination, and administration schedule

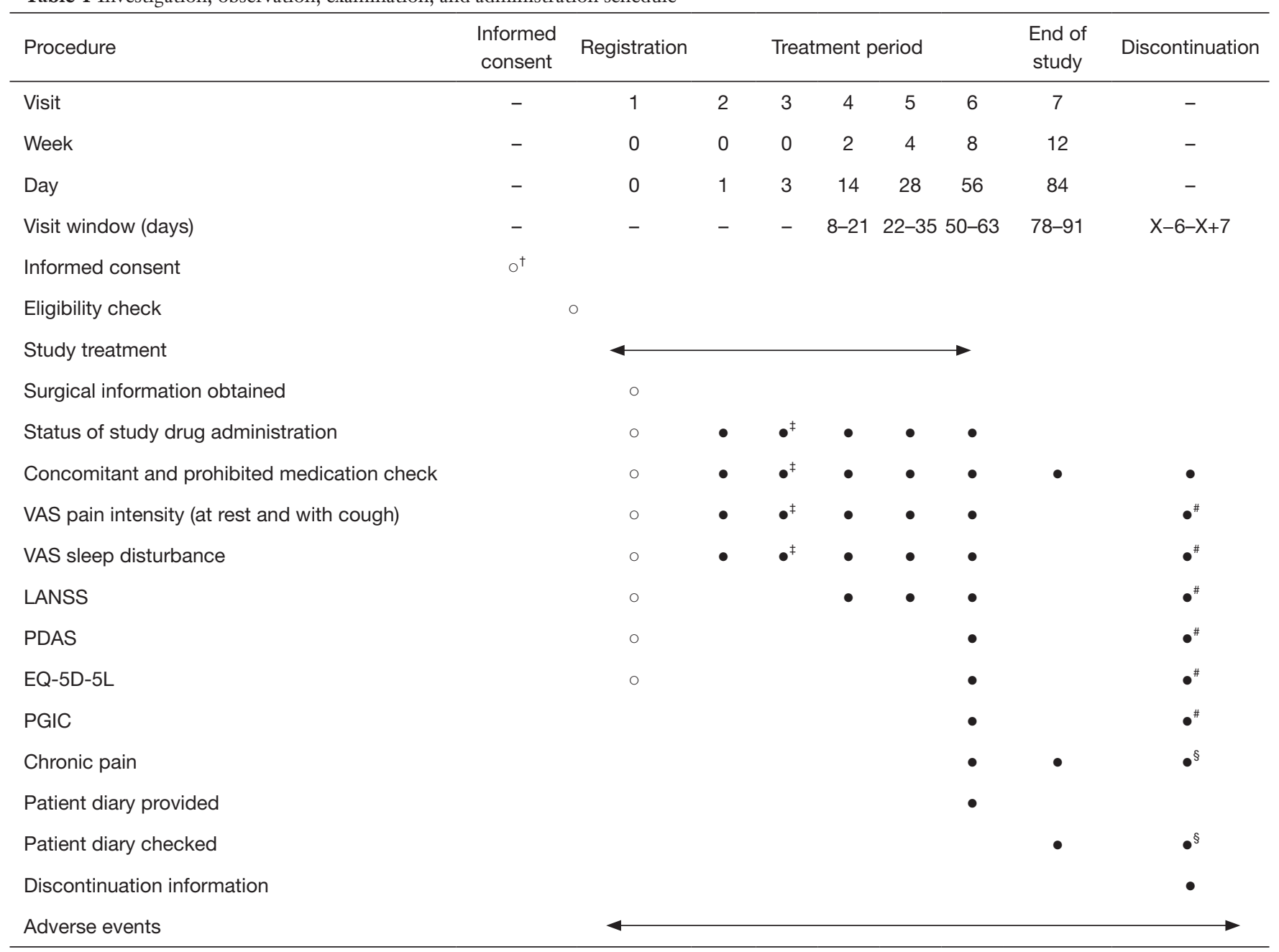

$\circ$ indicates items to be performed before the start of study treatment, and $\bullet$ indicates items to be performed after the start of study treatment. ${ }^{\dagger}$, informed consent will be obtained between the time of lung resection and the time of enrollment; ${ }^{\ddagger}$, Visit 3 should be performed as far as possible; \#, to be performed only if discontinuation occurs before Visit 6 ; ${ }^{\S}$, to be performed only if discontinuation occurs after Visit 6. Double-ended arrows indicates the duration of the study treatment and monitoring of adverse events. EQ-5D5L, EuroQol 5-dimension 5-level measure; LANSS, Leeds Assessment of Neuropathic Symptoms and Signs; PDAS, Pain Disability Assessment Scale; PGIC, Patient Global Impression of Change; VAS, visual analog scale.

measured during the observational period: pain intensity (using VAS at rest and while coughing); sleep disturbance (using VAS); Leeds Assessment of Neuropathic Symptoms and Signs (LANSS) score (37); Pain Disability Assessment Scale (PDAS) score (38); EuroQol 5-dimension 5-level (EQ-5D-5L) score (39); Patient Global Impression of Change (PGIC) (40); and adverse events (AEs) and adverse drug reactions elicited during physician interviews with patients. Treatment completion rates will be measured at study completion. In the case of $\mathrm{AE}$ onset, the investigator will assess each case individually and consider whether the patient should continue treatment at a maintained or reduced dose or if the patient should be discontinued from the study.

The primary study endpoint is the change in pain intensity from baseline to Week 8 , measured by VAS. Secondary endpoints are the proportion of patients with improvements of $\geq 30 \%$ and $\geq 50 \%$ from baseline at Week 8 in pain intensity (using VAS at rest); the percentage of patients with a LANSS score of $\geq 12$ at Weeks 2, 4, and 8; the change from baseline on various assessment scales, including VAS at rest (Day 1, Weeks 2 and 4), VAS while 
coughing (Day 1, Weeks 2, 4, and 8), PDAS (Week 8), EQ5D-5L (Week 8), VAS for sleep disturbance (Day 1, Weeks 2, 4, and 8), and PGIC (Week 8); and the prevalence of chronic pain at Weeks 8 and 12 in each treatment group.

\section{Sample size and statistical analyses}

With reference to a previous clinical study of pregabalin for postthoracotomy pain (26), the mean difference in change in VAS after 8 weeks between the conventional treatment group and the mirogabalin group was estimated to be 13.2 [standard deviation (SD) 22.5]. Thus, the number of patients needed to ensure $90 \%$ power at a twosided 5\% significance level would be 126 (63 patients per treatment group). Accounting for possible dropouts, the target sample size was set at 150 patients (i.e., 75 per group).

Baseline data for each treatment group will be summarized; categorical values will be summarized as frequency and percentage, and quantitative values will be summarized as the number of subjects, mean, $\mathrm{SD}$, minimum value, median value, and maximum value.

Primary efficacy analyses will be performed using a modified intent-to-treat (mITT) population, defined as all randomized patients who received at least one dose of the study drug. A linear mixed model for repeated measures (MMRM) will be applied to the primary endpoint data. Adjusted estimates of mean differences at Week 8 (mirogabalin combination group minus conventional treatment group) with their $95 \%$ confidence intervals and $\mathrm{P}$ values will be calculated. MMRM will include treatment group (mirogabalin, conventional treatment), time points (Day 1, Weeks 2, 4, and 8), and treatment-by-time interaction as fixed effects, VAS at enrollment (baseline) as covariates, and patient as a random effect. Summary statistics will be calculated for each time point and change from baseline by treatment group. For the secondary endpoints, frequency tables or summary statistics will be reported for the mITT population.

Sensitivity analyses will be performed using a perprotocol set, defined as all mITT patients who adhered to the study protocol. For the primary endpoint, VAS measurements at Week 8 will be imputed using the last observation carried forward method. Using analysis of covariance with baseline VAS as covariates, estimates of differences in adjusted means of VAS change from baseline to Week 8 (mirogabalin group minus conventional group) with their $95 \%$ confidence intervals and $\mathrm{P}$ values will be calculated. The same analysis will be performed when data handling is imputed using the baseline observation carried forward method.

Safety will be assessed in all enrolled patients who received at least one dose of the study drug. AEs will be coded using the medical dictionary for regulatory activities (MedDRA) version 23.1 or later. To calculate the completion rate for 8 weeks after thoracic surgery, the number of patients receiving the effective dose at Week 8 will be divided by the number of patients at the start of the initial dose (Week 1).

Statistical analyses will be performed using SAS version 9.4 or later (SAS Institute, Inc., Cary, NC, USA) and Microsoft Excel 2016 or later (Microsoft Corp., Redmond, WA, USA).

\section{Data management and study dissemination}

Patient information will be collected and stored in accordance with the Act on the Protection of Personal Information (Act No. 57 of 2003) and related notices. Appropriate anonymization will be applied. An electronic data capture system (CubeCDMS; CRScube APAC KK, Tokyo, Japan) will be used for data collation.

This research will be conducted in accordance with the Clinical Research Act (including the Enforcement Regulations of the Clinical Research Act and related notifications) and the protocol, and the study will be monitored by an independent Clinical Research Organization according to prespecified procedures and processes. Auditors will conduct document-based conformity investigation and field investigation independent of monitoring and ensure the reliability of research conduct and record-keeping. The investigators and participating medical organizations will facilitate the access of source documents to monitors and auditors when requested.

The sponsor, Daiichi Sankyo Co., Ltd., will provide the necessary funding for the conduct of this study and will be involved in designing the study plan, the data management plan, and the statistical analysis plan, in collecting safety information, and in overseeing the study and communicating important protocol modifications. However, the sponsor will not be directly involved in monitoring, data management, statistical analysis, or data auditing. All authors will have access to the final dataset. Study data will be published in the jRCT database and peerreviewed journals. 


\section{Acknowledgments}

The protocol will be presented at the $38^{\text {th }}$ Annual Meeting of the Japanese Association for Chest Surgery; May 20-21, 2021; Nagasaki, Japan. The authors would like to thank Masayuki Baba, MD, PhD of the Aomori Prefectural Central Hospital for supervising the pin-prick sensation tests conducted at registration. We also thank Sally-Anne Mitchell, PhD, of Edanz (www.edanz.com), for providing medical writing support, which was funded by Daiichi Sankyo Co., Ltd.

Funding: This study is supported by Daiichi Sankyo Co., Ltd.

\section{Footnote}

Reporting Checklist: The authors have completed the SPIRIT reporting checklist. Available at https://dx.doi. org/10.21037/jtd-21-741

Peer Review File: Available at https://dx.doi.org/10.21037/ jtd-21-741

Case Report Form: Available at https://dx.doi.org/10.21037/ jtd-21-741

Conflicts of Interest: All authors have completed the ICMJE uniform disclosure form (available at https://dx.doi. org/10.21037/jtd-21-741). T Miyazaki serves as an unpaid editorial board member of Journal of Thoracic Disease from Sep 2020 to Aug 2022. T Miyazaki and TN have received honoraria for lectures from Daiichi Sankyo Co., Ltd. KY, HT and KS are current employees of Daiichi Sankyo Co., Ltd. Daiichi Sankyo Co., Ltd. funded the research reflected in this manuscript, the medical writing support and the article processing charges.

Ethical Statement: The authors are accountable for all aspects of the work in ensuring that questions related to the accuracy or integrity of any part of the work are appropriately investigated and resolved. The study is being conducted in accordance with the Declaration of Helsinki (as revised in 2013). The study was approved by the Clinical Research Review Board of Nagasaki University (approval number CRB7180001), and informed consent will be obtained from all individual participants.

Open Access Statement: This is an Open Access article distributed in accordance with the Creative Commons Attribution-NonCommercial-NoDerivs 4.0 International License (CC BY-NC-ND 4.0), which permits the noncommercial replication and distribution of the article with the strict proviso that no changes or edits are made and the original work is properly cited (including links to both the formal publication through the relevant DOI and the license). See: https://creativecommons.org/licenses/by-nc-nd/4.0/.

\section{References}

1. Hardt J, Jacobsen C, Goldberg J, et al. Prevalence of chronic pain in a representative sample in the United States. Pain Med 2008;9:803-12.

2. US Department of Health and Human Services, Centers for Disdease Control and Prevention, National Center for Health Statistics. Health, United States, 2006, with chartbook on trends in the health of Americans. DHHS publication number 2006-1232; November 2006. Available online: https://www.cdc.gov/nchs/data/hus/hus06.pdf

3. Zelaya CE, Dahlhamer JM, Lucas JW, et al. Chronic Pain and High-impact Chronic Pain Among U.S. Adults, 2019. NCHS Data Brief 2020;(390):1-8.

4. Crombie IK, Davies HT, Macrae WA. Cut and thrust: antecedent surgery and trauma among patients attending a chronic pain clinic. Pain 1998;76:167-71.

5. Thapa P, Euasobhon P. Chronic postsurgical pain: current evidence for prevention and management. Korean J Pain 2018;31:155-73.

6. Werner MU, Kongsgaard UE. I. Defining persistent post-surgical pain: is an update required? $\mathrm{Br} \mathrm{J}$ Anaesth 2014;113:1-4.

7. Bayman EO, Brennan TJ. Incidence and severity of chronic pain at 3 and 6 months after thoracotomy: metaanalysis. J Pain 2014;15:887-97.

8. Dajczman E, Gordon A, Kreisman H, et al. Long-term postthoracotomy pain. Chest 1991;99:270-4.

9. Ochroch EA, Gottschalk A, Augostides J, et al. Long-term pain and activity during recovery from major thoracotomy using thoracic epidural analgesia. Anesthesiology 2002;97:1234-44.

10. Haanpää $M$, Attal N, Backonja $M$, et al. NeuPSIG guidelines on neuropathic pain assessment. Pain 2011;152:14-27.

11. Peng Z, Li H, Zhang C, et al. A retrospective study of chronic post-surgical pain following thoracic surgery: prevalence, risk factors, incidence of neuropathic component, and impact on qualify of life. PLoS One 
2014;9:e90014.

12. Haroutiunian S, Nikolajsen L, Finnerup NB, et al. The neuropathic component in persistent postsurgical pain: a systematic literature review. Pain 2013;154:95-102.

13. Miyazaki T, Sakai T, Tsuchiya T, et al. Assessment and follow-up of intercostal nerve damage after video-assisted thoracic surgery. Eur J Cardiothorac Surg 2011;39:1033-9.

14. Miyazaki T, Sakai T, Yamasaki N, et al. Chest tube insertion is one important factor leading to intercostal nerve impairment in thoracic surgery. Gen Thorac Cardiovasc Surg 2014;62:58-63.

15. Homma T, Shimada Y, Tanabe K, et al. Adverse factors and postoperative neuropathic pain in challenging video-assisted thoracoscopic surgery. Ann Palliat Med 2021;10:2849-58.

16. Fiorelli S, Cioffi L, Menna C, et al. Chronic Pain After Lung Resection: Risk Factors, Neuropathic Pain, and Quality of Life. J Pain Symptom Manage 2020;60:326-35.

17. Bayman EO, Parekh KR, Keech J, et al. A Prospective Study of Chronic Pain after Thoracic Surgery. Anesthesiology 2017;126:938-51.

18. Macrae WA. Chronic pain after surgery. Br J Anaesth 2001;87:88-98.

19. Sullivan EA. The role of the anesthesiologist in thoracic surgery: we can make a difference! J Cardiothorac Vasc Anesth 2009;23:761-5.

20. Gupta A, Bah M. NSAIDs in the Treatment of Postoperative Pain. Curr Pain Headache Rep 2016;20:62.

21. Sumitani M, Sakai T, Matsuda Y, et al. Executive summary of the Clinical Guidelines of Pharmacotherapy for Neuropathic Pain: second edition by the Japanese Society of Pain Clinicians. J Anesth 2018;32:463-78.

22. Bottiger BA, Esper SA, Stafford-Smith M. Pain management strategies for thoracotomy and thoracic pain syndromes. Semin Cardiothorac Vasc Anesth 2014;18:45-56.

23. Attal N, Bouhassira D. Pharmacotherapy of neuropathic pain: which drugs, which treatment algorithms? Pain 2015;156 Suppl 1:S104-14.

24. Finnerup NB, Attal N, Haroutounian S, et al. Pharmacotherapy for neuropathic pain in adults: a systematic review and meta-analysis. Lancet Neurol 2015;14:162-73.

25. Matsutani N, Dejima H, Takahashi Y, et al. Pregabalin reduces post-surgical pain after thoracotomy: a prospective, randomized, controlled trial. Surg Today 2015;45:1411-6.

26. Yoshimura N, Iida H, Takenaka M, et al. Effect of Postoperative Administration of Pregabalin for Post- thoracotomy Pain: A Randomized Study. J Cardiothorac Vasc Anesth 2015;29:1567-72.

27. Miyazaki T, Sakai T, Sato S, et al. Is early postoperative administration of pregabalin beneficial for patients with lung cancer?-randomized control trial. J Thorac Dis 2016;8:3572-9.

28. Tarlige (mirogabalin besilate) $2.5 \mathrm{mg}$ [drug information sheet]. Daiichi Sankyo Co., Ltd., Tokyo, Japan; revised March 2020. Available online: https://www.rad-ar.or.jp/ siori/english/kekka.cgi?n=41379

29. Burgess J, Javed S, Frank B, et al. Mirogabalin besylate in the treatment of neuropathic pain. Drugs Today (Barc) 2020;56:135-49.

30. Domon $Y$, Arakawa N, Inoue T, et al. Binding Characteristics and Analgesic Effects of Mirogabalin, a Novel Ligand for the $\alpha 2 \delta$ Subunit of VoltageGated Calcium Channels. J Pharmacol Exp Ther 2018;365:573-82.

31. Alyoubi RA, Alshareef AA, Aldughaither SM, et al. Efficacy and safety of mirogabalin treatment in patients with diabetic peripheral neuropathic pain: A systematic review and meta-analysis of randomised controlled trials. Int J Clin Pract 2021;75:e13744.

32. Baba M, Matsui N, Kuroha M, et al. Mirogabalin for the treatment of diabetic peripheral neuropathic pain: A randomized, double-blind, placebo-controlled phase III study in Asian patients. J Diabetes Investig 2019;10:1299-306.

33. Baba M, Matsui N, Kuroha M, et al. Long-term safety and efficacy of mirogabalin in Asian patients with diabetic peripheral neuropathic pain. J Diabetes Investig 2020;11:693-8.

34. Kato J, Matsui N, Kakehi Y, et al. Long-term safety and efficacy of mirogabalin in Asian patients with postherpetic neuralgia: Results from an open-label extension of a multicenter randomized, double-blind, placebo-controlled trial. Medicine (Baltimore) 2020;99:e21976.

35. Kato J, Matsui N, Kakehi Y, et al. Mirogabalin for the management of postherpetic neuralgia: a randomized, double-blind, placebo-controlled phase 3 study in Asian patients. Pain 2019;160:1175-85.

36. Finnerup NB, Haroutounian S, Kamerman P, et al. Neuropathic pain: an updated grading system for research and clinical practice. Pain 2016;157:1599-606.

37. Bennett M. The LANSS Pain Scale: the Leeds assessment of neuropathic symptoms and signs. Pain 2001;92:147-57.

38. Yamashiro K, Arimura T, Iwaki R, et al. A multidimensional measure of pain interference: reliability 
and validity of the pain disability assessment scale. Clin J Pain 2011;27:338-43.

39. EuroQol Group. EQ-5D instruments: About the 5-level EQ-5D. Available online: https://euroqol.org/eq-5dinstruments/eq-5d-5l-about/

40. Guy W. ECDEU assessment manual for

Cite this article as: Doi R, Miyazaki T, Tsuchiya T, Matsumoto K, Tomoshige K, Machino R, Mizoguchi S, Matsumoto T, Yamaguchi K, Takatsuna H, Shiosakai K, Nagayasu T. Mirogabalin treatment of postoperative neuropathic pain after thoracic surgery: study protocol for a multicenter, randomized, open-label, parallel-group, interventional trial. J Thorac Dis 2021;13(10):6062-6070. doi: $10.21037 /$ jtd-21-741 psychopharmacology, DHEW publication number ADM 76-338. US Government Printing Office, Washington, DC, USA; 1976. Available online: https://openlibrary.org/ books/OL24341821M/ECDEU_assessment_manual_for_ psychopharmacology 


\section{Supplementary}

Table S1 Details of participating medical institutions (to date)

\begin{tabular}{|c|c|c|}
\hline No. & Institution & Address \\
\hline 2 & National Hospital Organization Nagasaki Medical Center & 2-1001-1 Kubara, Omura, Nagasaki 856-8562, Japan \\
\hline 3 & $\begin{array}{l}\text { Local incorporated administrative agency Sasebo City } \\
\text { General Hospital }\end{array}$ & 9-3 Hirase-cho, Sasebo, Nagasaki 857-8511, Japan \\
\hline 5 & Ehime Prefectural Central Hospital & 83 Kasugamachi, Matsuyama, Ehime 790-0024, Japan \\
\hline 6 & Kumamoto University Hospital & 1-1-1 Honjo, Chuo Ward, Kumamoto 860-8556, Japan \\
\hline 7 & University of Miyazaki Hospital & 5200 Kihara, Kiyotakecho, Miyazaki 889-1692, Japan \\
\hline 8 & $\begin{array}{l}\text { Kagoshima University Graduate School of Medical and } \\
\text { Dental Sciences }\end{array}$ & 8-35-1 Sakuragaoka, Kagoshima 890-8544, Japan \\
\hline 10 & Japanese Red Cross Nagasaki Genbaku Hospital & 3-15 Morimachi, Nagasaki 852-8511, Japan \\
\hline 11 & Niigata University Medical and Dental Hospital & $\begin{array}{l}\text { 754, Ichibancho, Asahimachidori, Chuo-ku, Niigata-shi, Niigata 951-8520, } \\
\text { Japan }\end{array}$ \\
\hline 12 & University of Tsukuba Hospital & 2-1-1 Amakubo, Tsukuba, Ibaraki 305-8576, Japan \\
\hline 13 & Tokyo Medical University Hospital & 6-7-1 Nishi-Shinjuku, Shinjuku-ku, Tokyo 160-0023, Japan \\
\hline 14 & Fukuoka University Hospital & 7-45-1 Nanakuma, Jonan-ku, Fukuoka 814-0180, Japan \\
\hline
\end{tabular}

